Supplement of

\title{
Aerosol pH and its influencing factors in Beijing
}

Jing Ding ${ }^{2,1}$, Pusheng Zhao ${ }^{1,3}$, Jie Su ${ }^{1}$, Qun Dong1, and Xiang Du' ${ }^{1}$

${ }^{1}$ Institute of Urban Meteorology, China Meteorological Administration, Beijing 100089, China

${ }^{2}$ State Environmental Protection Key Laboratory of Urban Ambient Air Particulate Matter

Pollution Prevention and Control, College of Environmental Science and Engineering, Nankai University, Tianjin 300071, China

${ }^{3}$ Max Planck Institute for Chemistry, Mainz 55128, Germany

*Corresponding to: P. S. Zhao (pszhao@ium.cn) 


\section{Supplementary materials}
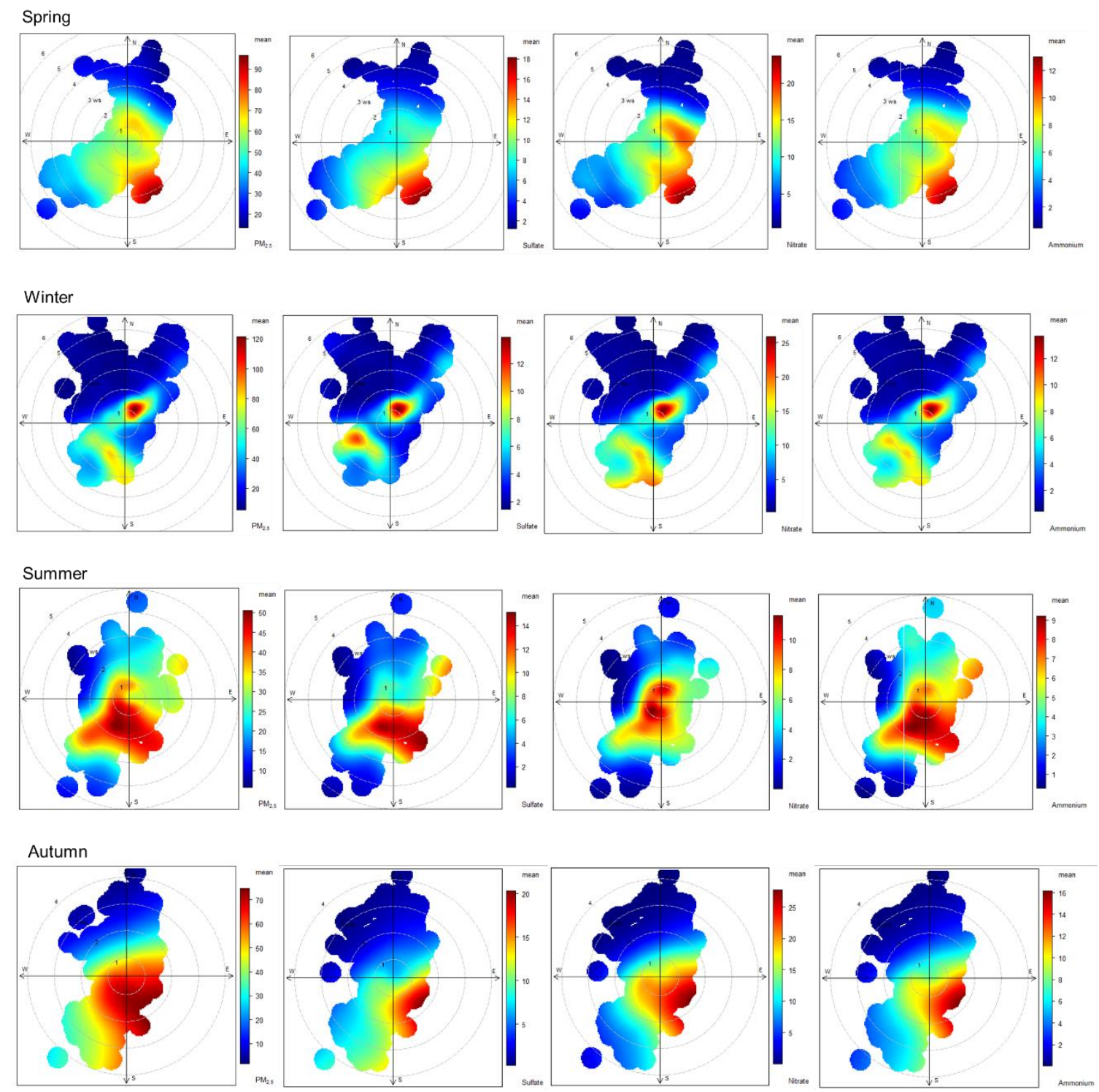

Fig. S1 Wind dependence map of $\mathrm{PM}_{2.5}, \mathrm{SO}_{4}{ }^{2-}, \mathrm{NO}_{3}{ }^{-}, \mathrm{NH}_{4}{ }^{+}$over four seasons. In each picture, the shaded contour indicates the average of variables for varying wind speeds (radial direction) and wind directions (transverse direction). 

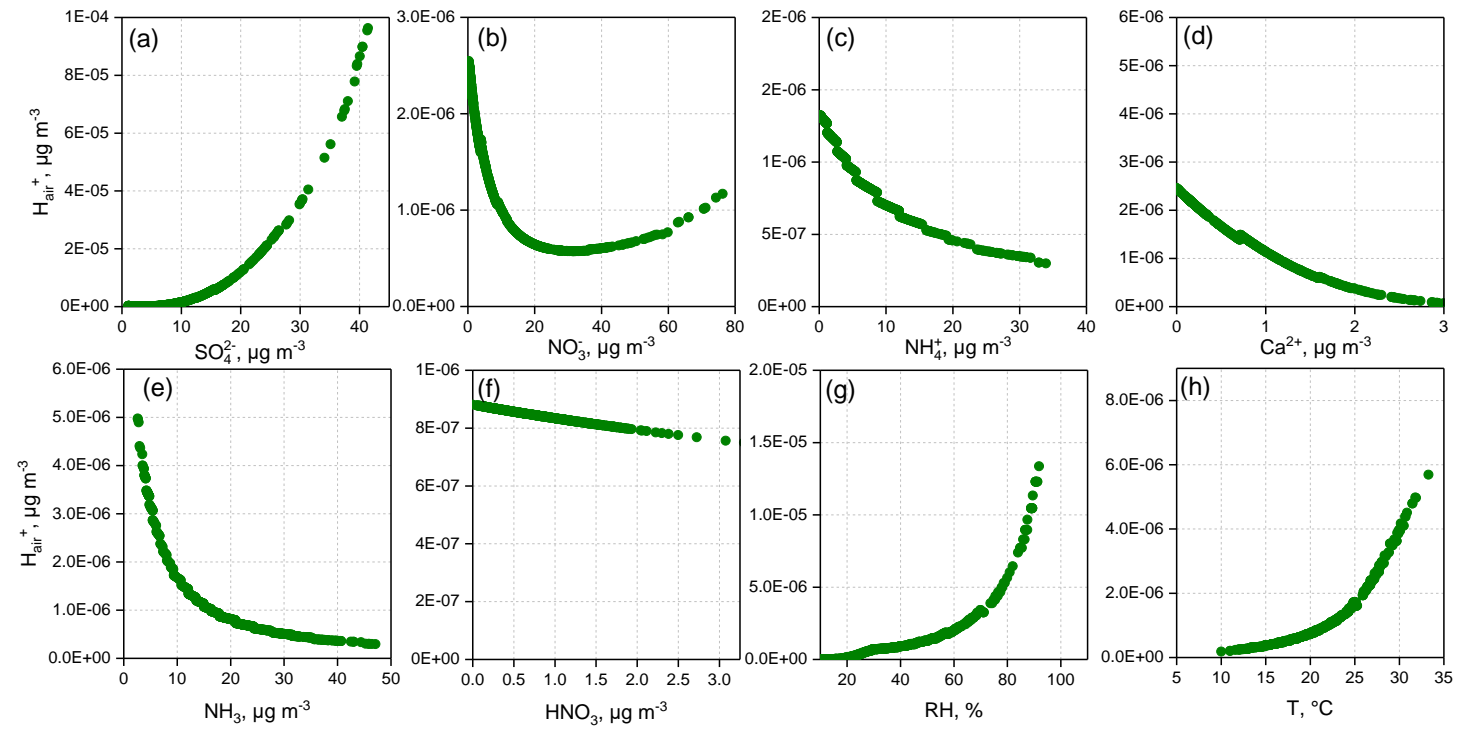

Fig. S2. Sensitivities of chemical components $\left(\mathrm{NO}_{3}{ }^{-}, \mathrm{SO}_{4}{ }^{2-}, \mathrm{NH}_{4}{ }^{+}, \mathrm{Ca}^{2+}\right)$, precursor gases $\left(\mathrm{NH}_{3}\right.$, $\mathrm{HNO}_{3}$ ) as well as meteorological parameters (RH, T) to ALWC in spring.
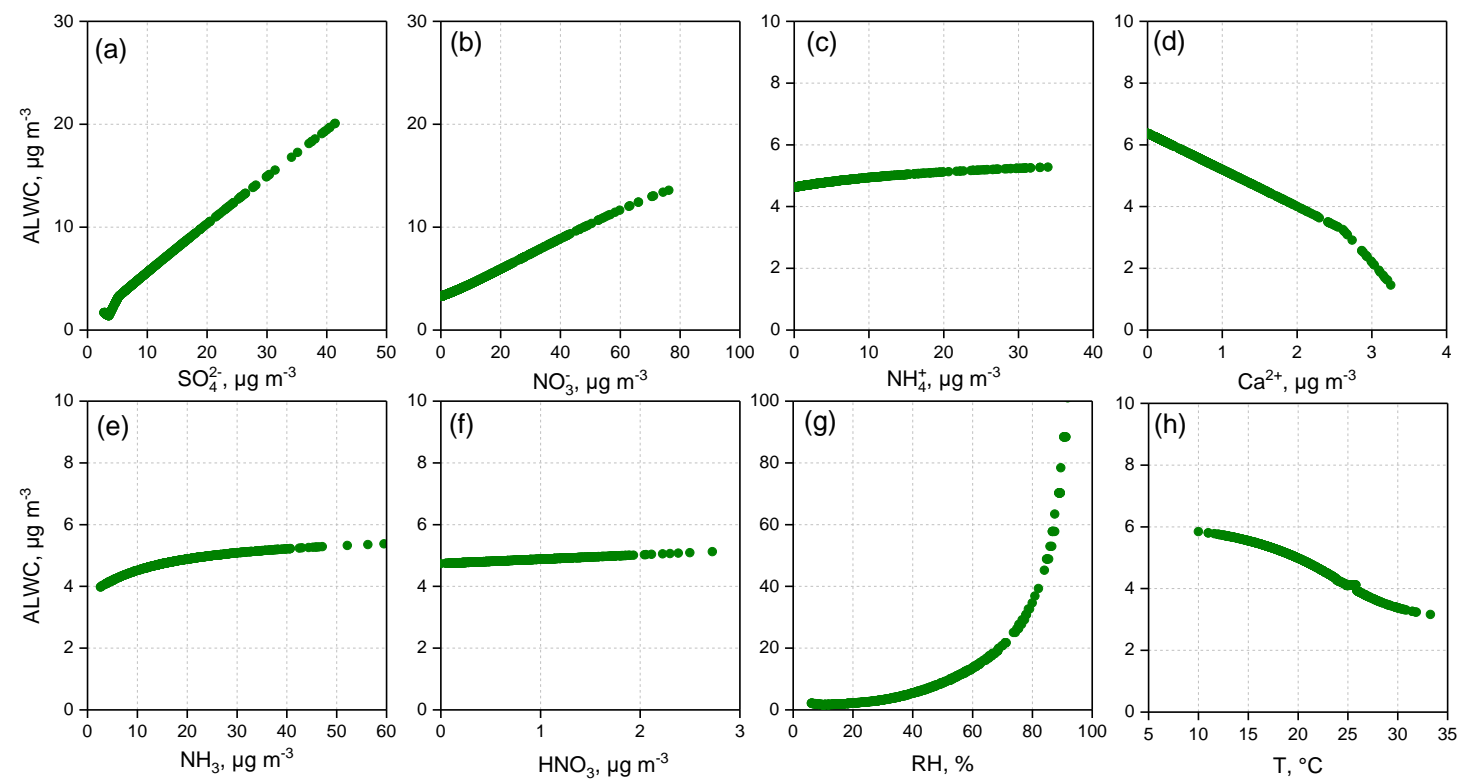

Fig. S3. Sensitivities of chemical components $\left(\mathrm{NO}_{3}{ }^{-}, \mathrm{SO}_{4}{ }^{2-}, \mathrm{NH}_{4}{ }^{+}, \mathrm{Ca}^{2+}\right)$, precursor gases $\left(\mathrm{NH}_{3}\right.$, $\mathrm{HNO}_{3}$ ) as well as meteorological parameters (RH, T) to $\mathrm{H}_{\text {air }}{ }^{+}$in spring. 

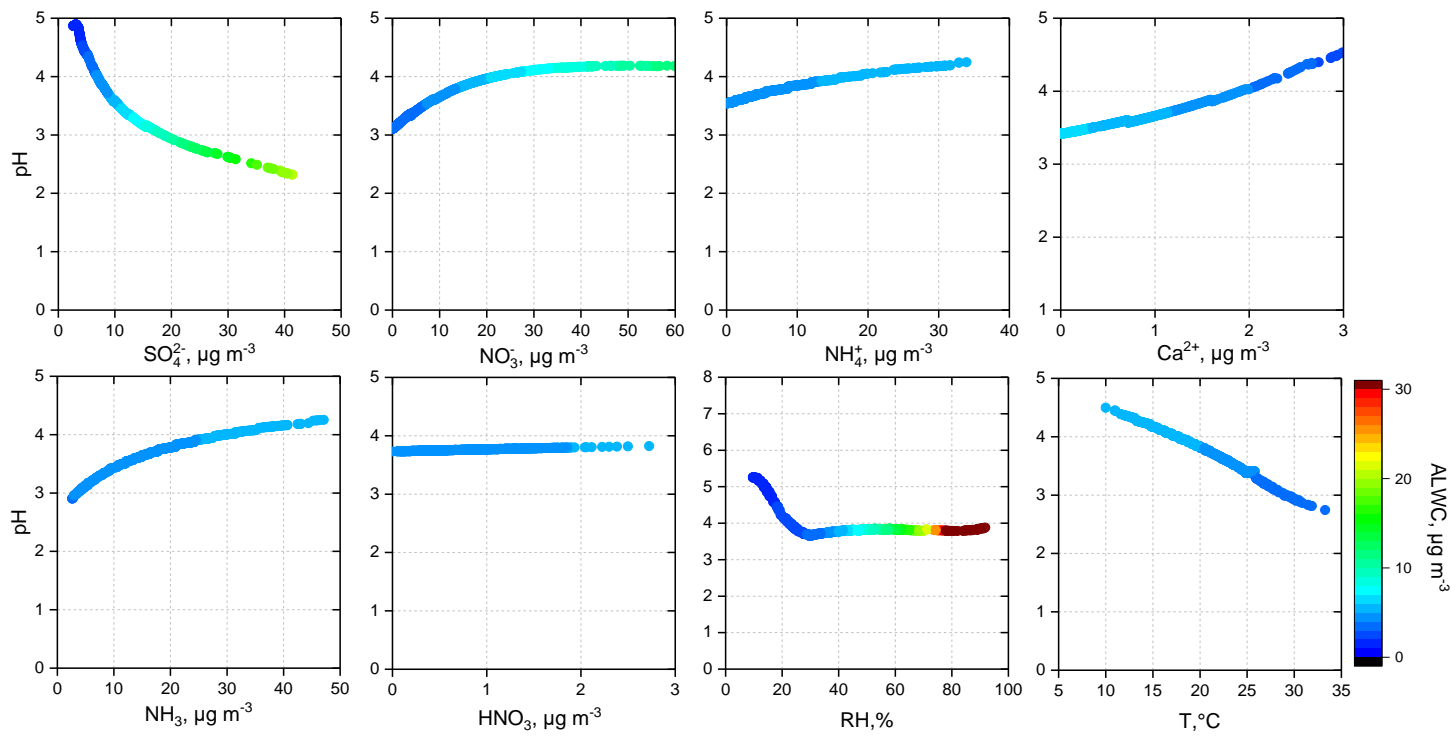

Fig. S4. Sensitivities of chemical components $\left(\mathrm{NO}_{3}{ }^{-}, \mathrm{SO}_{4}{ }^{2-}, \mathrm{NH}_{4}{ }^{+}, \mathrm{Ca}^{2+}\right)$, precursor gases $\left(\mathrm{NH}_{3}\right.$, $\mathrm{HNO}_{3}$ ) as well as meteorological parameters $(\mathrm{RH}, \mathrm{T})$ to $\mathrm{pH}$ in spring.
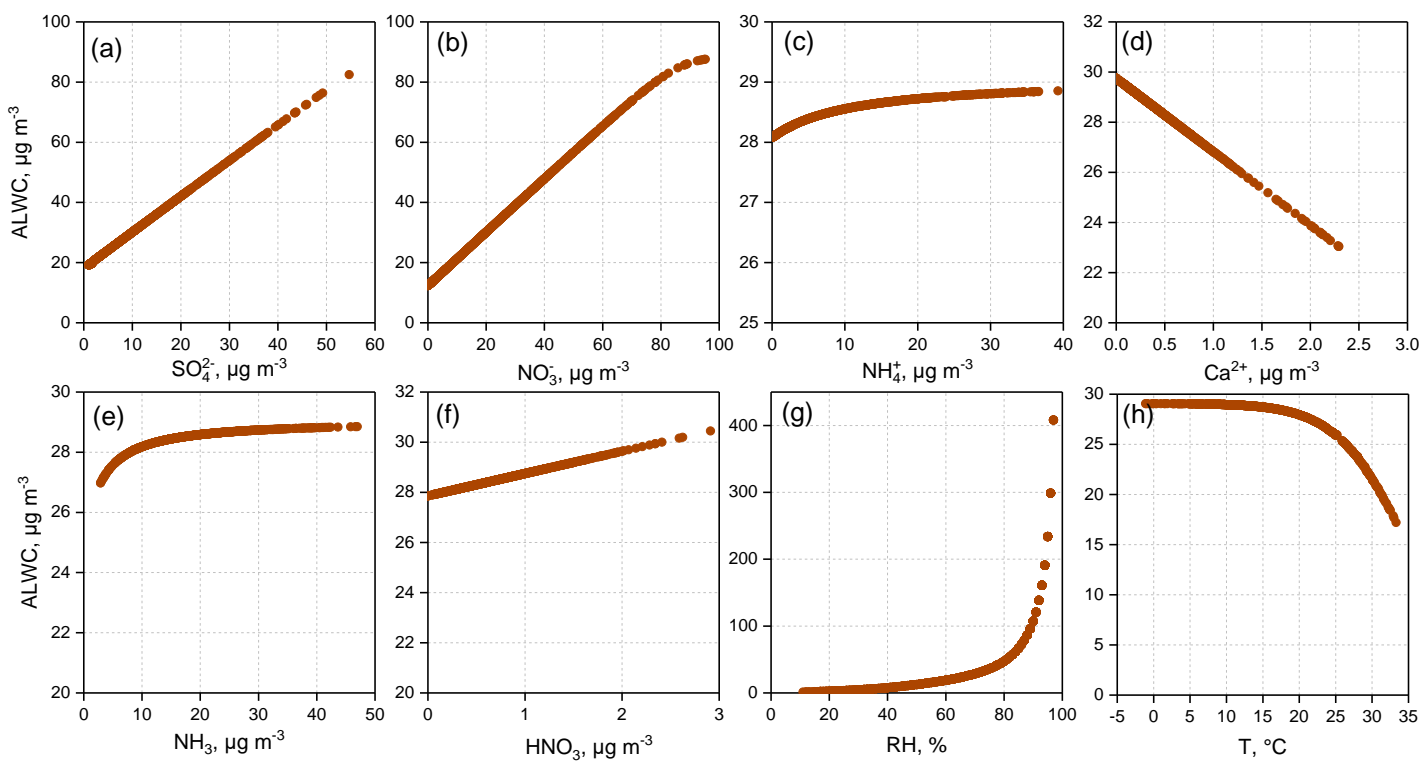

Fig. S5. Sensitivities of chemical components $\left(\mathrm{NO}_{3}{ }^{-}, \mathrm{SO}_{4}{ }^{2-}, \mathrm{NH}_{4}{ }^{+}, \mathrm{Ca}^{2+}\right)$, precursor gases $\left(\mathrm{NH}_{3}\right.$, $\left.\mathrm{HNO}_{3}\right)$ as well as meteorological parameters (RH, T) to ALWC autumn. 

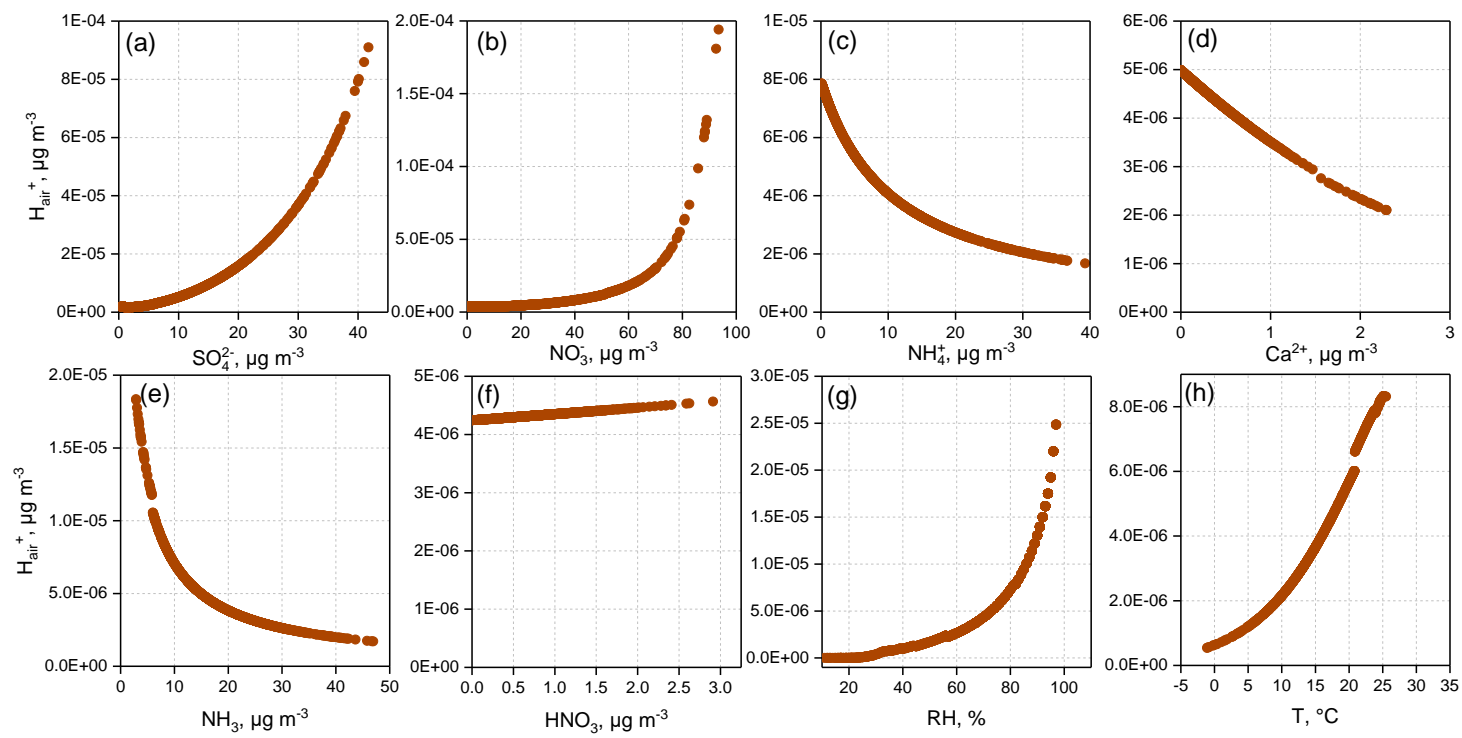

Fig. S6. Sensitivities of chemical components $\left(\mathrm{NO}_{3}{ }^{-}, \mathrm{SO}_{4}{ }^{2-}, \mathrm{NH}_{4}{ }^{+}, \mathrm{Ca}^{2+}\right)$, precursor gases $\left(\mathrm{NH}_{3}\right.$, $\left.\mathrm{HNO}_{3}\right)$ as well as meteorological parameters $(\mathrm{RH}, \mathrm{T})$ to $\mathrm{H}_{\text {air }}{ }^{+}$in autumn.
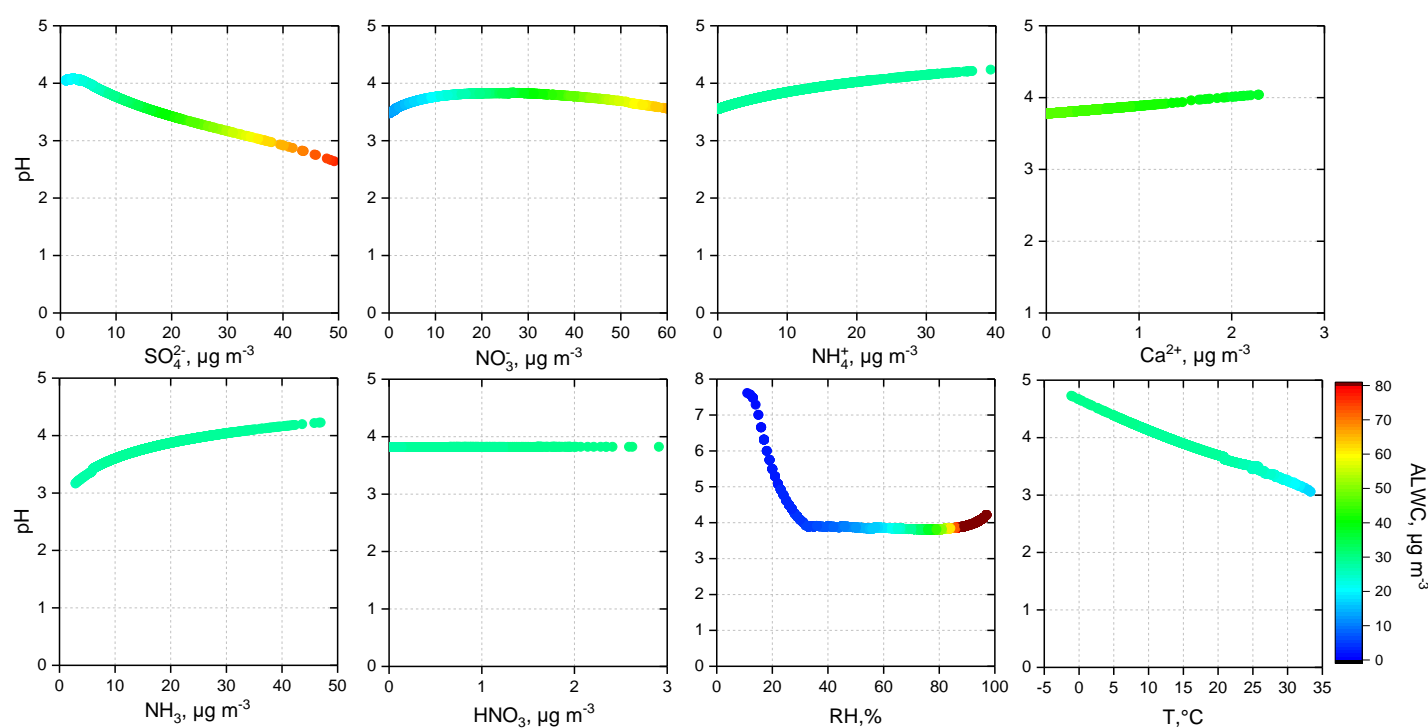

Fig. S7. Sensitivities of chemical components $\left(\mathrm{NO}_{3}{ }^{-}, \mathrm{SO}_{4}{ }^{2-}, \mathrm{NH}_{4}{ }^{+}, \mathrm{Ca}^{2+}\right)$, precursor gases $\left(\mathrm{NH}_{3}\right.$, $\mathrm{HNO}_{3}$ ) as well as meteorological parameters (RH, T) to $\mathrm{pH}$ in autumn. 

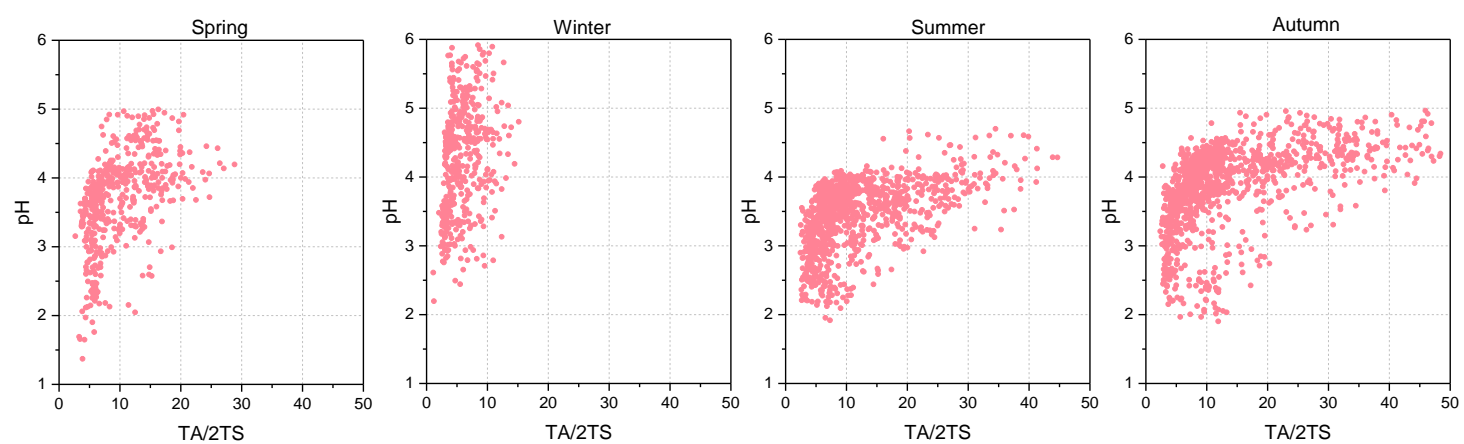

Fig. S8. The predicted aerosol $\mathrm{pH}$ versus measured TA/2TS ratio $\left(\right.$ mole mole $\left.{ }^{-1}\right)$ over four seasons.

Data are restricted to aerosol $\mathrm{pH}$ in the range $1 \sim 5$ for spring, summer and autumn as well as $1 \sim 6$ in winter according to the aerosol $\mathrm{pH}$ frequency distribution. 